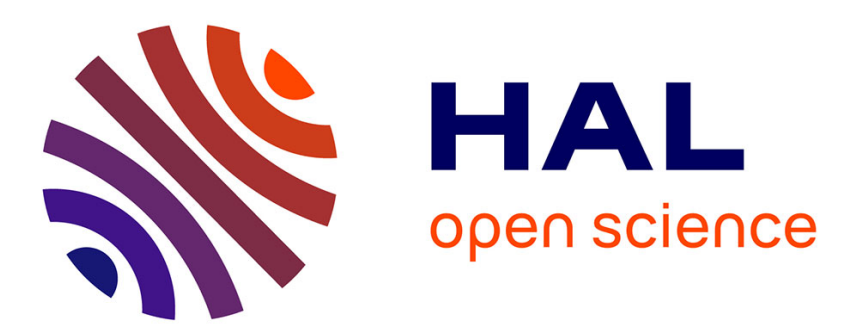

\title{
Non-central limit theorem for the cubic variation of a class of selfsimilar stochastic processes
}

\author{
Khalifa Es-Sebaiy, Ciprian A. Tudor
}

\section{To cite this version:}

Khalifa Es-Sebaiy, Ciprian A. Tudor. Non-central limit theorem for the cubic variation of a class of selfsimilar stochastic processes. SIAM Theory of Probability and its Applications, 2010, 55 (3), pp.1-23. hal-00432690

\section{HAL Id: hal-00432690 https://hal.science/hal-00432690}

Submitted on 16 Nov 2009

HAL is a multi-disciplinary open access archive for the deposit and dissemination of scientific research documents, whether they are published or not. The documents may come from teaching and research institutions in France or abroad, or from public or private research centers.
L'archive ouverte pluridisciplinaire $\mathbf{H A L}$, est destinée au dépôt et à la diffusion de documents scientifiques de niveau recherche, publiés ou non, émanant des établissements d'enseignement et de recherche français ou étrangers, des laboratoires publics ou privés. 


\title{
Non-central limit theorem for the cubic variation of a class of selfsimilar stochastic processes
}

\author{
Khalifa Es-Sebaiy ${ }^{1}$ Ciprian A. Tudor ${ }^{1,2}$ \\ ${ }^{1}$ SAMOS/MATISSE, Centre d'Economie de La Sorbonne, \\ Université de Panthéon-Sorbonne Paris 1, \\ 90, rue de Tolbiac, 75634 Paris Cedex 13, France. \\ Khalifa.Es-Sebaiy@malix.univ-paris1.fr \\ ${ }^{2}$ Laboratoire Paul Painlevé, U.F. R. de Mathématiques \\ Université de Lille 1 , \\ Cité Scientifique, 59655 Villeneuve d'Ascq Cedex, France. \\ tudor@math.univ-lille1.fr
}

November 9, 2009

\begin{abstract}
By using multiple Wiener-Itô stochastic integrals, we study the cubic variation of a class of selfsimilar stochastic processes with stationary increments (the Rosenblatt process with selfsimilarity order $\left.H \in\left(\frac{1}{2}, 1\right)\right)$. This study is motivated by statistical purposes. We prove that this renormalized cubic variation satisfies a non-central limit theorem and its limit is (in the $L^{2}(\Omega)$ sense) still the Rosenblatt process.
\end{abstract}

2000 AMS Classification Numbers: 60G15, 60G35, 60H05, 94A05.

Key words: multiple stochastic integrals, selfsimilar processes, Rosenblatt process,fractional Brownian motion, non-central limit theorem, Malliavin calculus.

\section{Introduction}

The self-similarity property for a stochastic process means that scaling of time is equivalent to an appropriate scaling of space. That is, a process $\left(Y_{t}\right)_{t \geq 0}$ is selfsimilar of order $H>0$ if for all $c>0$ the processes $\left(Y_{c t}\right)_{t \geq 0}$ and $\left(c^{H} Y_{t}\right)_{t \geq 0}$ have the same finite dimensional distributions. The selfsimilar processes are of interest for various applications, such as economics, internet traffic of hydrology. The fractional Brownian motion (fBm) is the usual candidate to model phenomena in which the selfsimilarity property can be observed from the empirical data. Recall that the fractional Brownian motion is a centered Gaussian process with covariance function $R^{H}(t, s)=\frac{1}{2}\left(t^{2 H}+s^{2 H}-|t-s|^{2 H}\right)$. The parameter $H \in(0,1)$ characterizes almost all the important properties of the process. The $\mathrm{fBm}$ can be also defined as the only Gaussian process which is selfsimilar with stationary increments. In some models the gaussianity assumption 
could be not plausible and in this case one needs to use a different selfsimilar process with stationary increments to model the phenomena. Natural candidates are the Hermite processes: these stochastic processes appear as limits in the so-called Non-Central Limit Theorem (see [3], [7], [18], [10]). In contrast with the classical Central Limit Theorem, the non-central limit theorem deals with sequences of dependent random variable whose renomalized sum converges in some situations to a non gaussian distribution. For a complete exposition of limit theorems in probability theory, we refer to [9] or [16]. Except the Gaussian character, these Hermite processes have the same property as the $\mathrm{fBm}$ with Hurst parameter $H>\frac{1}{2}$ : selfsimilarity, stationarity of increments, Hölder continuous path, long -range dependence. While the fractional Brownian motion can be expressed as a Wiener integral with respect to the standard Wiener process, the Hermite process of order $q \geq 2$ is a $q$ iterated integral of a deterministic function with $q$ variables with respect to the Brownian motion. The Rosenblatt process is obtained in the particular case $q=2$. It will be properly defined in Section 2. This processes have been recently studied by several authors (see [2], [4], [13], [11], [12], [19], [20]).

The Hurst parameter $H$ characterizes all the important properties of a Hermite process, as seen above. Therefore, estimating $H$ properly is of the utmost importance. Several statistics have been introduced to this end, such as wavelets, $k$-variations, variograms, maximum likelihood estimators, or spectral methods. Information on these various approaches can be found in the book of Beran [1].

One of the most popular methods to estimate the selfsimilarity order for stochastic process is based on the study of their variations. The $p$ - variation of a process $\left(X_{t}\right)_{t \in[0,1]}$ is defined as the limit of the sequence (sometimes the absolute value of the increment is used in the definition)

$$
V^{p, N}(X)=\frac{1}{N}\left[\sum_{i=0}^{N-1} \frac{\left(X_{\frac{i+1}{N}}-X_{\frac{i}{N}}\right)^{p}}{\mathbf{E}\left(X_{\frac{i+1}{N}}-X_{\frac{i}{N}}\right)^{p}}-1\right] .
$$

There exists a direct connection between the behavior of the variations and the convergence of an estimator for the selfsimilarity order based of these variation (see [6], [20]); basically if there renormalized variation satisfies a central limit theorem then the estimator satisfies a central limit theorem and this fact is very useful for statistical aspects.

In a recent paper ([20]) the quadratic variation of the Rosenblatt process $\left(Z_{t}^{(H)}\right)_{t \in[0,1]}$ with selfsimilarity order $H \in\left(\frac{1}{2}, 1\right)$ has been studied. The following facts happen: the normalized sequence $N^{1-H} V^{2, N}\left(Z^{(H)}\right)$ satisfies a non-central limit theorem, it converges in $L^{2}$ to the Rosenblatt random variable $Z_{1}^{(H)}$. From this, we can construct an estimator for $H$ whose behavior is still non-normal. This situation is somehow not good for statistical applications because one always prefers the estimators which are asymptotically normal. To have normal estimators we need to define some adjusted variations (as in [20]).

In the fractional Brownian motion case the well-known non-normality of the quadratic variation when $H \in\left(\frac{3}{4}, 1\right)$ can be avoided by using "longer filters" (that means, replacing the increments $X_{\frac{i+1}{N}}-X_{\frac{i}{N}}$ by $X_{\frac{i+1}{N}}-2 X_{\frac{i}{N}}+X_{\frac{i-1}{N}}$ ) or higher order variations (choosing a bigger $p$ ). In this work we will consider the second choice (the first choice has been treated in the paper [5]): we replace the quadratic variation by the cubic variation for the Rosenblatt processes to see what happens and if it is possible to find a Gaussian distribution as law of the renormalized cubic variation. In the fractional Brownian motion case, this has no sense because the third moment of a centered Gaussian random variable is zero. We use the Wiener chaos expansion for the statistics $V^{3, N}\left(Z^{(H)}\right)$ and we will decompose it in several terms in 
the Wiener chaoses 2,4 and 6. As in other cases ([20], [4]) the second chaos term is dominant and it has to be renormalized by $N^{1-H}$ to have a non-trivial limit. We note that the rate of convergence $N^{1-H}$ is the same as for quadratic variation, so there no gain for the speed and moreover the limit is again, modulo a constant, a Rosenblatt random variable with index $H$ (only the constant is changing). This property has been called in [4] the reproduction property of the Rosenblatt process because its variations generates again Rosenblatt random variable as limits. We conjecture that the same property holds true for the $p$ variations.

The organization of our paper is as follows. Section 2 contains the presentation of the basic tools that we will need throughout the paper: multiple Wiener-Itô integrals and their basic properties, the definition of the Rosenblatt process and its characteristics. In Section 3 we estimate the mean square of the cubic variation of the Rosenblatt process and we give its normalization and finally in Section 4 we prove a non-central limit theorem for the renormalized cubic variation.

\section{Preliminaries}

\subsection{Multiple stochastic integrals}

In this paragraph we describe the basic elements of calculus on Wiener chaos. Let $\left(W_{t}\right)_{t \in[0,1]}$ be a classical Wiener process on a standard Wiener space $(\Omega, \mathcal{F}, \mathbf{P})$. If $f \in L^{2}\left([0,1]^{n}\right)$ with $n \geq 1$ integer, we introduce the multiple Wiener-Itô integral of $f$ with respect to $W$. We refer to [14] for a detailed exposition of the construction and the properties of multiple Wiener-Itô integrals.

Let $f \in \mathcal{S}_{m}$ be an elementary functions with $m$ variables that can be written as

$$
f=\sum_{i_{1}, \ldots, i_{m}} c_{i_{1}, \ldots i_{m}} 1_{A_{i_{1}} \times \ldots \times A_{i_{m}}}
$$

where the coefficients satisfy $c_{i_{1}, \ldots i_{m}}=0$ if two indices $i_{k}$ and $i_{l}$ are equal and the sets $A_{i} \in \mathcal{B}([0,1])$ are disjoints. For a such step function $f$ we define

$$
I_{m}(f)=\sum_{i_{1}, \ldots, i_{m}} c_{i_{1}, \ldots i_{m}} W\left(A_{i_{1}}\right) \ldots W\left(A_{i_{m}}\right)
$$

where we put $W([a, b])=W_{b}-W_{a}$. It can be seen that the application $I_{n}$ constructed above from $\mathcal{S}$ to $L^{2}(\Omega)$ is an isometry on $\mathcal{S}$, i.e.

$$
\mathbf{E}\left[I_{n}(f) I_{m}(g)\right]=n !\langle f, g\rangle_{L^{2}\left([0,1]^{n}\right)} \text { if } m=n
$$

and

$$
\mathbf{E}\left[I_{n}(f) I_{m}(g)\right]=0 \text { if } m \neq n \text {. }
$$

It also holds that

$$
I_{n}(f)=I_{n}(\tilde{f})
$$

where $\tilde{f}$ denotes the symmetrization of $f$ defined by $\tilde{f}\left(x_{1}, \ldots, x_{n}\right)=\frac{1}{n !} \sum_{\sigma \in S_{n}} f\left(x_{\sigma(1)}, \ldots, x_{\sigma(n)}\right)$.

Since the set $\mathcal{S}_{n}$ is dense in $L^{2}\left([0,1]^{n}\right)$ for every $n \geq 1$ the mapping $I_{n}$ can be extended to an isometry from $L^{2}\left([0,1]^{n}\right)$ to $L^{2}(\Omega)$ and the above properties hold true for this extension. Note also that $I_{n}$ can be viewed as an iterated stochastic integral

$$
I_{n}(f)=n ! \int_{0}^{1} \int_{0}^{t_{n}} \ldots \int_{0}^{t_{2}} f\left(t_{1}, \ldots, t_{n}\right) d W_{t_{1}} \ldots d W_{t_{n}} ;
$$


here the integrals are of Itô type; this formula is easy to show for elementary f's, and follows for general $f \in L^{2}\left([0,1]^{n}\right)$ by a density argument.

The product for two multiple integrals can be expanded into a sum of multiple integrals (see [14]): if $f \in L^{2}\left([0,1]^{n}\right)$ and $g \in L^{2}\left([0,1]^{m}\right)$ are symmetric functions, then it holds that

$$
I_{n}(f) I_{m}(g)=\sum_{l=0}^{m \wedge n} l ! C_{m}^{l} C_{n}^{l} I_{m+n-2 l}\left(f \otimes_{l} g\right)
$$

where the contraction $f \otimes_{l} g$ belongs to $L^{2}\left([0,1]^{m+n-2 l}\right)$ for $l=0,1, \ldots, m \wedge n$ and it is given by

$$
\begin{aligned}
&\left(f \otimes_{l} g\right)\left(s_{1}, \ldots, s_{n-l}, t_{1}, \ldots, t_{m-l}\right) \\
&= \int_{[0,1]} f\left(s_{1}, \ldots, s_{n-l}, u_{1}, \ldots, u_{l}\right) g\left(t_{1}, \ldots, t_{m-l}, u_{1}, \ldots, u_{l}\right) d u_{1} \ldots d u_{l} .
\end{aligned}
$$

When $l=0$, we will denote, throughout this paper, by $f \otimes g:=f \otimes_{0} g$.

\subsection{The Rosenblatt process}

The Rosenblatt process $\left(Z^{(H)}(t)\right)_{t \in[0,1]}$ appears as a limit in the so-called Non Central Limit Theorem (see [7], [18], [10]). It is not a Gaussian process and can be defined through its representation as double iterated integral with respect to a standard Wiener process (see [19]). More precisely, the Rosenblatt process with self-similarity order $H \in\left(\frac{1}{2}, 1\right)$ is defined by

$$
Z_{t}^{(H)}:=\int_{0}^{t} \int_{0}^{t} L_{t}\left(y_{1}, y_{2}\right) d W_{y_{1}} d W_{y_{2}}
$$

where $\left(W_{t}, t \in[0,1]\right)$ is a Brownian motion,

$$
L_{t}^{H}\left(y_{1}, y_{2}\right):=L_{t}\left(y_{1}, y_{2}\right)=d(H) 1_{[0, t]}\left(y_{1}\right) 1_{[0, t]}\left(y_{2}\right) \int_{y_{1} \vee y_{2}}^{t} \frac{\partial K^{H^{\prime}}}{\partial u}\left(u, y_{1}\right) \frac{\partial K^{H^{\prime}}}{\partial u}\left(u, y_{2}\right) d u
$$

with

$$
H^{\prime}:=\frac{H+1}{2} \quad \text { and } \quad d(H):=\frac{1}{H+1}\left(\frac{H}{2(2 H-1)}\right)^{-\frac{1}{2}} .
$$

and with $K^{H}$ the standard kernel defined in (7) appearing in the Wiener integral representation of the fBm (for $t>s$ and $H>\frac{1}{2}$ )

$$
K^{H}(t, s):=c_{H} s^{\frac{1}{2}-H} \int_{s}^{t}(u-s)^{H-\frac{3}{2}} u^{H-\frac{1}{2}} d u
$$

with $c_{H}=\left(\frac{H(2 H-1)}{\beta\left(2-2 H, H-\frac{1}{2}\right)}\right)^{\frac{1}{2}}$ and $\beta(\cdot, \cdot)$ the beta function. The derivative of $K^{H}$ is

$$
\frac{\partial K^{H}}{\partial t}(t, s):=\partial_{1} K^{H}(t, s)=c_{H}\left(\frac{s}{t}\right)^{\frac{1}{2}-H}(t-s)^{H-\frac{3}{2}} .
$$

The two parameters function $L_{t}$ given by (6) will be called the kernel of the Rosenblatt process. The following key relation in crucial in our calculation and it will repeatedly used in the paper

$$
\int_{0}^{u \wedge v} \partial_{1} K^{H^{\prime}}(u, y) \partial_{1} K^{H^{\prime}}(v, y) d y=a(H)|u-v|^{2 H^{\prime}-2}
$$

with $a(H)=H^{\prime}\left(2 H^{\prime}-1\right)$. Among the main properties of the Rosenblatt process, we recall 
- it is $H$-self-similar in the sense that for any $c>0,\left(Z_{c t}^{(H)}\right)={ }^{(d)}\left(c^{H} Z_{t}^{(H)}\right)$, where $"={ }^{(d)}$ " means equivalence of all finite dimensional distributions;

- it has stationary increments, that is, the joint distribution of $\left(Z_{t+h}^{(H)}-Z_{h}^{(H)}, t \in[0,1]\right)$ is independent of $h>0$.

- $\mathbf{E}\left(\left|Z_{t}^{(H)}\right|^{p}\right)<\infty$ for any $p>0$, and $Z^{(H)}$ has the same variance and covariance than a standard fractional Brownian motion with parameter $H$.

- the Rosenblatt process is Hölder continuous, of order $\delta<H$. This can easily obtained by the Kolmogorov continuity criterium.

\section{Renormalization of the cubic variation}

\subsection{Estimation of the mean square}

We will study in this paragraph the cubic variation of the Rosenblatt process obtained by putting $p=3$ in (1)

$$
V^{3, N}=\frac{1}{N} \sum_{i=0}^{N-1}\left(\frac{\left(Z_{\frac{i+1}{N}}^{(H)}-Z_{\frac{i}{N}}^{(H)}\right)^{3}}{\mathbf{E}\left(Z_{\frac{i+1}{N}}^{(H)}-Z_{\frac{i}{N}}^{(H)}\right)^{3}}-1\right)
$$

Note that this expression is immaterial is the case of the fractional Brownian motion because the third moment of a centered Gaussian random variable is zero. By denoting for $=1, \ldots, N$

$$
f_{i, N}=L_{\frac{i+1}{N}}^{(H)}-L_{\frac{i}{N}}^{(H)}
$$

we obtain $Z_{\frac{i+1}{N}}^{(H)}-Z_{\frac{i}{N}}^{(H)}=I_{2}\left(f_{i, N}\right)$ where $I_{2}$ is a multiple integral of order 2 as defined in Section 2.1 and then

$$
V^{3, N}=\frac{1}{N} \sum_{i=0}^{N-1}\left(\frac{\left(I_{2}\left(f_{i, N}\right)\right)^{3}}{\mathbf{E}\left(I_{2}\left(f_{i, N}\right)\right)^{3}}-1\right) .
$$

By using the product formula for multiple Wiener-Itô integrals (3), for any function $f \in$ $L^{2}\left([0,1]^{2}\right)$ symmetric,

$$
\begin{aligned}
& I_{2}(f)^{3} \\
= & I_{6}((f \tilde{\otimes} f) \otimes f)+8 I_{4}\left((f \tilde{\otimes} f) \otimes_{1} f\right)+4 I_{4}\left(\left(f \otimes_{1} f\right) \otimes f\right) \\
& +12 I_{2}\left((f \tilde{\otimes} f) \otimes_{2} f\right)+16 I_{2}\left(\left(f \otimes_{1} f\right) \otimes_{1} f\right)+2\langle f, f\rangle_{L^{2}\left([0,1]^{2}\right)} I_{2}(f)+8\left\langle\left(f \otimes_{1} f\right), f\right\rangle_{L^{2}\left([0,1]^{2}\right)} .
\end{aligned}
$$

Here and in the sequel $f \tilde{\otimes} f$ denotes the symmetrization of the function $f \otimes f$ which is not necessary symmetric even if $f$ is symmetric. Applying this to $f=f_{i, N}$ we obtain

$$
\left(I_{2}\left(f_{i, N}\right)\right)^{3}=8\left(f_{i, N} \otimes_{1} f_{i, N}\right) \otimes_{2} f_{i, N}+I_{2}\left(g_{i, N}\right)+4 I_{4}\left(h_{i, N}\right)+I_{6}\left(\left(f_{i, N} \tilde{\otimes} f_{i, N}\right) \otimes f_{i, N}\right)(11)
$$

Here we used the following notation

$$
g_{i, N}=2\left\|f_{i, N}\right\|_{L^{2}}^{2} f_{i, N}+12\left(f_{i, N} \tilde{\otimes} f_{i, N}\right) \otimes_{2} f_{i, N}+16\left(f_{i, N} \otimes_{1} f_{i, N}\right) \otimes_{1} f_{i, N}
$$


and

$$
h_{i, N}=2\left(f_{i, N} \tilde{\otimes} f_{i, N}\right) \otimes_{1} f_{i, N}+f_{i, N} \otimes\left(f_{i, N} \otimes_{1} f_{i, N}\right):=h_{i, N}^{(1)}+h_{i, N}^{(2)} .
$$

Note that $g_{i, N} \in L^{2}\left([0,1]^{2}\right)$ and $h_{i, N} \in L^{2}\left([0,1]^{4}\right)$. On the other hand, we can simplify a little bit the above expressions since

$$
\left(f_{i, N} \tilde{\otimes} f_{i, N}\right) \otimes_{2} f_{i, N}=\frac{1}{3}\left\|f_{i, N}\right\|_{L^{2}}^{2} f_{i, N}+\frac{2}{3}\left(f_{i, N} \otimes_{1} f_{i, N}\right) \otimes_{1} f_{i, N} .
$$

Hence the kernel of the second chaos term can be written as

$$
g_{i, N}=6\left\|f_{i, N}\right\|_{L^{2}}^{2} f_{i, N}+24\left(f_{i, N} \otimes_{1} f_{i, N}\right) \otimes_{1} f_{i, N} .
$$

We start with the following lemma where we compute the cubic mean of the increment of the Rosenblatt process. We already observe a significant difference from the Gaussian case: this cubic mean is not zero.

Lemma 1 Let $\left(Z_{t}^{(H)}\right)_{t \in[0,1]}$ be a Rosenblatt process with selfsimilarity index $H \in\left(\frac{1}{2}, 1\right)$. Then, for every $s, t \in[0,1]$

$$
\mathbf{E}\left(Z_{t}^{(H)}-Z_{s}^{(H)}\right)^{3}=C(H)|t-s|^{3 H}
$$

where

$$
C(H)=8 a(H)^{3} d(H)^{3} \int_{[0,1]^{3}}\left(\left|u-v\left\|u-u^{\prime}\right\| v-u^{\prime}\right|\right)^{2 H^{\prime}-2} d u d u^{\prime} d v .
$$

Proof. Let us denote by

$$
f_{s, t}(x, y)=L_{t}(x, y)-L_{s}(x, y)
$$

where $L$ is the kernel of the Rosenblatt process given by (6) and $x, y \in[0,1]$. We will have, by using relation (9),

$$
\begin{aligned}
\left(f_{s, t} \otimes_{1} f_{s, t}\right)(x, y)= & \int_{0}^{1} f_{s, t}(x, z) f_{s, t}(y, z) d z \\
= & d(H)^{2} a(H)\left(1_{[0, t]}(x, y) \int_{x}^{t} \int_{y}^{t} \partial_{1} K^{H^{\prime}}(u, x) \partial_{1} K^{H^{\prime}}(v, y)|u-v|^{2 H^{\prime}-2} d v d u\right. \\
& -1_{[0, t]}(x) 1_{[0, s]}(y) \int_{x}^{t} \int_{y}^{s} \partial_{1} K^{H^{\prime}}(u, x) \partial_{1} K^{H^{\prime}}(v, y)|u-v|^{2 H^{\prime}-2} d v d u \\
& -1_{[0, s]}(x) 1_{[0, t]}(y) \int_{x}^{s} \int_{y}^{t} \partial_{1} K^{H^{\prime}}(u, x) \partial_{1} K^{H^{\prime}}(v, y)|u-v|^{2 H^{\prime}-2} d v d u \\
& \left.+1_{[0, s]}(x, y) \int_{x}^{s} \int_{y}^{s} \partial_{1} K^{H^{\prime}}(u, x) \partial_{1} K^{H^{\prime}}(v, y)|u-v|^{2 H^{\prime}-2} d v d u\right) .
\end{aligned}
$$

The computation of the cubic mean of a multiple integral in the second chaos (11) implies

$$
\mathbf{E}\left(Z_{t}^{(H)}-Z_{s}^{(H)}\right)^{3}=8\left\langle f_{s, t} \otimes_{1} f_{s, t}, f_{s, t}\right\rangle_{L^{2}\left([0,1]^{2}\right)} .
$$

We compute, by (9) 


$$
\begin{aligned}
\left\langle f_{s, t} \otimes_{1} f_{s, t}, f_{s, t}\right\rangle_{L^{2}\left([0,1]^{2}\right)} & =\int_{[0,1]^{2}}\left(f_{s, t} \otimes_{1} f_{s, t}\right)(x, y) f_{s, t}(x, y) d x d y \\
& =d(H)^{3} a(H)^{3} \int_{s}^{t} \int_{s}^{t} \int_{s}^{t}\left(\left|u-v\left\|u-u^{\prime}\right\| v-u^{\prime}\right|\right)^{2 H^{\prime}-2} d u d u^{\prime} d v .
\end{aligned}
$$

By the change of variables $\bar{u}=\frac{u-s}{t-s}$ we will transform the integrals on $[s, t]$ into integrals from 0 to 1 . We immediately obtain the relation (14).

To calculate $\mathbf{E}\left(V^{3, N}\right)^{2}$ we apply the above result and we obtain

$$
\begin{aligned}
\mathbf{E}\left[\left(I_{2}\left(f_{i, N}\right)\right)^{3}\right] & =8\left(f_{i, N} \otimes_{1} f_{i, N}\right) \otimes_{2} f_{i, N} \\
& =8 d(H)^{3} a(H)^{3} \int_{I_{i}} \int_{I_{i}} \int_{I_{i}} d y_{1} d y_{2} d y_{3}\left(\left|y_{1}-y_{2}\right| \cdot\left|y_{2}-y_{3}\right| \cdot\left|y_{3}-y_{1}\right|\right)^{2 H^{\prime}-2} \\
& =8 \frac{d(H)^{3} a(H)^{3}}{N^{6 H^{\prime}-3}} \int_{[0,1]^{3}} d y_{1} d y_{2} d y_{3}\left(\left|y_{1}-y_{2}\right| \cdot\left|y_{2}-y_{3}\right| \cdot\left|y_{3}-y_{1}\right|\right)^{2 H^{\prime}-2} \\
& =C(H) N^{-\left(6 H^{\prime}-3\right)}=C(H) N^{-3 H} .
\end{aligned}
$$

where $a(H)=\frac{H(H+1)}{2}$ and $C(H)$ is defined in (15).

We can write the expression of the statistics $V_{N}$ as follows

$$
\begin{aligned}
V^{3, N} & =\frac{1}{C(H) N^{1-3 H}} \sum_{i=0}^{N-1}\left(\left(I_{2}\left(f_{i, N}\right)\right)^{3}-\mathbf{E}\left(I_{2}\left(f_{i, N}\right)\right)^{3}\right) \\
& =\frac{1}{C(H) N^{1-3 H}} \sum_{i=0}^{N-1}\left(I_{2}\left(g_{i, N}\right)+4 I_{4}\left(h_{i, N}\right)+I_{6}\left(\left(f_{i, N} \tilde{\otimes} f_{i, N}\right) \otimes f_{i, N}\right)\right) .
\end{aligned}
$$

We prove next the following renormalization result.

Proposition 2 Let $V^{3, N}$ the cubic variation statistics of the Rosenblatt process. Then

$$
\mathbf{E}\left(N^{1-H} V^{3, N}\right)^{2} \rightarrow_{N \rightarrow \infty} \bar{C}(H)
$$

where $\bar{C}(H):=C(H)^{2} C_{0}(H)$ with

$$
C_{0}(H)=\left(9+36 C^{\prime}(H) H(2 H-1)+144\left[C^{\prime}(H) H(2 H-1)\right]^{2}\right) .
$$


Proof. The isometry property of multiple Wiener-Itô integrals and relation (16) imply

$$
\begin{aligned}
& \mathbf{E}\left(V^{3, N}\right)^{2}=\frac{1}{N^{2}\left(\mathbf{E}\left(I_{2}\left(f_{i, N}\right)\right)^{3}\right)^{2}} \sum_{i, j=0}^{N-1}\left[\mathbf{E}\left(I_{2}\left(g_{i, N}\right) I_{2}\left(g_{j, N}\right)\right)+16 \mathbf{E}\left(I_{4}\left(h_{i, N}\right) I_{4}\left(h_{j, N}\right)\right)\right. \\
& \left.+\mathbf{E}\left(I_{6}\left(\left(f_{i, N} \tilde{\otimes} f_{i, N}\right) \otimes f_{i, N}\right) I_{6}\left(\left(f_{j, N} \tilde{\otimes} f_{j, N}\right) \otimes f_{j, N}\right)\right)\right] \\
& =\frac{1}{C(H)^{2} N^{2-6 H}}\left[\sum_{i, j=0}^{N-1} 2 !\left\langle g_{i, N}, g_{j, N}\right\rangle_{L^{2}\left([0,1]^{2}\right)}\right. \\
& +\sum_{i, j=0}^{N-1} 4 ! \times 16\left\langle\widetilde{h_{i, N}}, \widetilde{h_{j, N}}\right\rangle_{L^{2}\left([0,1]^{4}\right)} \\
& \left.+\sum_{i, j=0}^{N-1} 6 !\left\langle\left(f_{i, N} \tilde{\otimes} f_{i, N}\right) \otimes f_{i, N},\left(f_{j, N} \tilde{\otimes} f_{j, N}\right) \otimes f_{j, N}\right\rangle_{L^{2}\left([0,1]^{6}\right)}\right] \\
& :=\frac{1}{C(H)^{2} N^{2-6 H}}\left[A_{N}^{(2)}+B_{N}^{(4)}+D_{N}^{(6)}\right] .
\end{aligned}
$$

We use the notation $A_{N}^{(2)}$ to indicate that this term comes from the estimation of the second chaos summand of $V^{3, N}$, and similarly for the terms $B_{N}^{(4)}$ and $D_{N}^{(6)}$. We will try to estimate the all the three terms above to see which is the dominant term of $V_{N}$.

Estimation of the term $A_{N}^{(2)}$. We start by calculating $A_{N}^{(2)}$. Taking into account the expression of the second chaos kernel $g_{i, N}(12)$

$$
\begin{aligned}
& A_{N}^{(2)}:= \sum_{i, j=0}^{N-1} 2<g_{i, N}, g_{j, N}>_{L^{2}\left([0,1]^{2}\right)} \\
&=2 \sum_{i, j=0}^{N-1}\left[36\left\|f_{i, N}\right\|_{L^{2}\left([0,1]^{2}\right)}^{2}\left\|f_{j, N}\right\|_{L^{2}\left([0,1]^{2}\right)}^{2}\left\langle f_{i, N}, f_{j, N}\right\rangle_{L^{2}\left([0,1]^{2}\right)}\right. \quad+144\left\|f_{i, N}\right\|_{L^{2}\left([0,1]^{2}\right)}^{2}\left\langle f_{i, N},\left(f_{j, N} \otimes_{1} f_{j, N}\right) \otimes_{1} f_{j, N}\right\rangle_{L^{2}\left([0,1]^{2}\right)} \\
&\left.\quad+(24)^{2}\left\langle\left(f_{i, N} \otimes_{1} f_{i, N}\right) \otimes_{1} f_{i, N},\left(f_{j, N} \otimes_{1} f_{j, N}\right) \otimes_{1} f_{j, N}\right\rangle_{L^{2}\left([0,1]^{2}\right)} \cdot\right] \\
&:=2\left(36 A_{1, N}^{(2)}+144 A_{2, N}^{(2)}+(24)^{2} A_{3, N}^{(2)}\right)
\end{aligned}
$$

Let us evaluate the term $A_{1, N}^{(2)}$, we have

$$
2 !\left\|f_{i, N}\right\|_{L^{2}\left([0,1]^{2}\right)}^{2}=\mathbf{E}\left|Z_{\frac{i+1}{N}}^{(H)}-Z_{\frac{i}{N}}^{(H)}\right|^{2}=N^{-2 H} .
$$

Furthermore

$$
2\left\langle f_{i, N}, f_{j, N}\right\rangle_{L^{2}\left([0,1]^{2}\right)}=\mathbf{E}\left(Z_{\frac{i+1}{N}}^{(H)}-Z_{\frac{i}{N}}^{(H)}\right)\left(Z_{\frac{j+1}{N}}^{(H)}-Z_{\frac{j}{N}}^{(H)}\right)
$$


Hence

$$
\begin{aligned}
A_{1, N}^{(2)} & =\sum_{i, j=0}^{N-1}\left[\left\|f_{i, N}\right\|_{L^{2}\left([0,1]^{2}\right)}^{2}\left\|f_{j, N}\right\|_{L^{2}\left([0,1]^{2}\right)}^{2}\left\langle f_{i, N}, f_{j, N}\right\rangle_{L^{2}\left([0,1]^{2}\right)}\right] \\
& =\frac{1}{8} N^{-4 H} \sum_{i, j=0}^{N-1} \mathbf{E}\left(Z_{\frac{i+1}{N}}^{(H)}-Z_{\frac{i}{N}}^{(H)}\right)\left(Z_{\frac{j+1}{N}}^{(H)}-Z_{\frac{j}{N}}^{(H)}\right)=\frac{1}{8} N^{-4 H}
\end{aligned}
$$

because $\mathbf{E} \sum_{i, j=0}^{N-1}\left(Z_{\frac{i+1}{N}}^{(H)}-Z_{\frac{i}{N}}^{(H)}\right)\left(Z_{\frac{j+1}{N}}^{(H)}-Z_{\frac{j}{N}}^{(H)}\right)=\mathbf{E}\left(Z_{1}^{(H)}\right)^{2}=1$ and we have

$$
\lim _{N \rightarrow \infty} N^{4 H} A_{1, N}^{(2)}=\frac{1}{8}
$$

We evaluate $A_{3, N}^{(2)}$. Note that

$$
\begin{aligned}
& d(H)^{-2} a(H)^{-1}\left(f_{i, N} \otimes_{1} f_{i, N}\right)(x, y) \\
= & 1_{\left[0, \frac{i}{N}\right]}^{\otimes 2}(x, y) \int_{I_{i}} \int_{I_{i}} \partial_{1} K^{H^{\prime}}\left(u_{1}, x\right) \partial_{1} K^{H^{\prime}}\left(u_{2}, y\right)\left|u_{1}-u_{2}\right|^{2 H^{\prime}-2} d u_{2} d u_{1} \\
& +1_{\left[0, \frac{i}{N}\right]}(x) 1_{I_{i}}(y) \int_{I_{i}} \int_{y}^{\frac{i+1}{N}} \partial_{1} K^{H^{\prime}}\left(u_{1}, x\right) \partial_{1} K^{H^{\prime}}\left(u_{2}, y\right)\left|u_{1}-u_{2}\right|^{2 H^{\prime}-2} d u_{2} d u_{1} \\
& +1_{\left[0, \frac{i}{N}\right]}(y) 1_{I_{i}}(x) \int_{x}^{\frac{i+1}{N}} \int_{I_{i}} \partial_{1} K^{H^{\prime}}\left(u_{1}, x\right) \partial_{1} K^{H^{\prime}}\left(u_{2}, y\right)\left|u_{1}-u_{2}\right|^{2 H^{\prime}-2} d u_{2} d u_{1} \\
& +1_{I_{i}}(x) 1_{I_{i}}(y) \int_{x}^{\frac{i+1}{N}} \int_{y}^{\frac{i+1}{N}} \partial_{1} K^{H^{\prime}}\left(u_{1}, x\right) \partial_{1} K^{H^{\prime}}\left(u_{2}, y\right)\left|u_{1}-u_{2}\right|^{2 H^{\prime}-2} d u_{2} d u_{1} .
\end{aligned}
$$

Sometimes its useful to use to following compressed expression

$$
\left(f_{i, N} \otimes_{1} f_{i, N}\right)(x, y)=d(H)^{2} a(H) 1_{\left[0, \frac{i+1}{N}\right]}^{\otimes 2}(x, y) \int_{I_{i}} \int_{I_{i}} \partial_{1} K^{H^{\prime}}\left(u_{1}, x\right) \partial_{1} K^{H^{\prime}}\left(u_{2}, y\right)\left|u_{1}-u_{2}\right|^{2 H^{\prime}-2} d u_{2} d u_{1}
$$

and

$$
\begin{aligned}
& d(H)^{-1} f_{i, N}(x, z) \\
= & 1_{\left[0, \frac{i}{N}\right]}^{\otimes 2}(x, z) \int_{I_{i}} \partial_{1} K^{H^{\prime}}\left(u_{3}, x\right) \partial_{1} K^{H^{\prime}}\left(u_{3}, z\right) d u_{3}+1_{\left[0, \frac{i}{N}\right]}(x) 1_{I_{i}}(z) \int_{z}^{\frac{i+1}{N}} \partial_{1} K^{H^{\prime}}\left(u_{3}, x\right) \partial_{1} K^{H^{\prime}}\left(u_{3}, z\right) d u_{3} \\
& +1_{\left[0, \frac{i}{N}\right]}(z) 1_{I_{i}}(x) \int_{x}^{\frac{i+1}{N}} \partial_{1} K^{H^{\prime}}\left(u_{3}, x\right) \partial_{1} K^{H^{\prime}}\left(u_{3}, z\right) d u_{3} \\
& +1_{I_{i}}(x) 1_{I_{i}}(z) \int_{I_{i}} \int_{I_{i}} \partial_{1} K^{H^{\prime}}\left(u_{3}, x\right) \partial_{1} K^{H^{\prime}}\left(u_{3}, z\right) d u_{3}
\end{aligned}
$$

or otherwise

$$
f_{i, N}(x, z)=d(H) 1_{\left[0, \frac{i+1}{N}\right]}^{\otimes 2}(x, z) \int_{I_{i}} \partial_{1} K^{H^{\prime}}\left(u_{3}, x\right) \partial_{1} K^{H^{\prime}}\left(u_{3}, z\right) d u_{3}
$$


Therefore

$$
\begin{aligned}
& \left(\left(f_{i, N} \otimes_{1} f_{i, N}\right) \otimes_{1} f_{i, N}\right)(y, z)=d(H)^{3} a(H)^{2}\left(1_{\left[0, \frac{i+1}{N}\right]}^{\otimes 2}(y, z)\right. \\
& \left.\times \int_{I_{i}} \int_{I_{i}} \int_{I_{i}}\left(\left|u_{1}-u_{2}\right|\left|u_{1}-u_{3}\right|\right)^{2 H^{\prime}-2} \partial_{1} K^{H^{\prime}}\left(u_{2}, y\right) \partial_{1} K^{H^{\prime}}\left(u_{3}, z\right) d u_{3} d u_{2} d u_{1}\right) .
\end{aligned}
$$

The norm has a nicer expression. Using the change of variables $\bar{u}=\left(u-\frac{i}{N}\right) N$ (which is now usual and it can be used systematically) we have

$$
\begin{aligned}
& \left\langle\left(f_{i, N} \otimes_{1} f_{i, N}\right) \otimes_{1} f_{i, N},\left(f_{j, N} \otimes_{1} f_{j, N}\right) \otimes_{1} f_{j, N}\right\rangle_{L^{2}\left([0,1]^{2}\right)} \\
= & d(H)^{6} a(H)^{6} \int_{I_{i}^{3}} d u_{1} d u_{2} d u_{3} \int_{I_{j}^{3}} d v_{1} d v_{2} d v_{3} \\
& \left|u_{1}-u_{2}\right|^{2 H^{\prime}-2}\left|u_{1}-u_{3}\right|^{2 H^{\prime}-2}\left|v_{1}-v_{2}\right|^{2 H^{\prime}-2}\left|v_{1}-v_{3}\right|^{2 H^{\prime}-2}\left|u_{2}-v_{2}\right|^{2 H^{\prime}-2}|| u_{3}-\left.v_{3}\right|^{2 H^{\prime}-2} \\
= & \frac{d(H)^{6} a(H)^{6}}{N^{12 H^{\prime}-6}} \int_{[0,1]^{3}} \int_{[0,1]^{3}}\left|v_{1}-v_{2}\right|^{2 H^{\prime}-2}\left|v_{2}-v_{3}\right|^{2 H^{\prime}-2}\left|v_{3}-v_{4}+i-j\right|^{2 H^{\prime}-2} \\
& \quad \times\left|v_{4}-v_{5}\right|^{2 H^{\prime}-2}\left|v_{5}-v_{6}\right|^{2 H^{\prime}-2}\left|v_{6}-v_{1}+j-i\right|^{2 H^{\prime}-2} d v_{1} \ldots d v_{6} .
\end{aligned}
$$

The rate of convergence of all terms presents in this proof comes actually from how many product $|u-v|^{2 H^{\prime}-2}$ with $u \in I_{i}$ and $v \in I_{j}$ we have. Hence

$$
\begin{aligned}
& A_{3, N}^{(2)}=\frac{d(H)^{6} a(H)^{6}}{N^{12 H^{\prime}-6}} \sum_{i, j=1}^{N} \int_{[0,1]^{3}} \int_{[0,1]^{3}}\left|v_{1}-v_{2}\right|^{2 H^{\prime}-2}\left|v_{2}-v_{3}\right|^{2 H^{\prime}-2}\left|v_{3}-v_{4}+i-j\right|^{2 H^{\prime}-2} \\
& \times\left|v_{4}-v_{5}\right|^{2 H^{\prime}-2}\left|v_{5}-v_{6}\right|^{2 H^{\prime}-2}\left|v_{6}-v_{1}+j-i\right|^{2 H^{\prime}-2} d v_{1} \ldots d v_{6} \\
& =\frac{2 d(H)^{6} a(H)^{6}}{N^{12 H^{\prime}-6}} \sum_{i>j=1}^{N} \int_{[0,1]^{3}} \int_{[0,1]^{3}}\left|v_{1}-v_{2}\right|^{2 H^{\prime}-2}\left|v_{2}-v_{3}\right|^{2 H^{\prime}-2}\left|v_{3}-v_{4}+i-j\right|^{2 H^{\prime}-2} \\
& \times\left|v_{4}-v_{5}\right|^{2 H^{\prime}-2}\left|v_{5}-v_{6}\right|^{2 H^{\prime}-2}\left|v_{1}-v_{6}+i-j\right|^{2 H^{\prime}-2} d v_{1} \ldots d v_{6} \\
& =\frac{2 d(H)^{6} a(H)^{6}}{N^{12 H^{\prime}-6}} \sum_{k=0}^{N-1}(N-k) \int_{[0,1]^{3}} \int_{[0,1]^{3}}\left|v_{1}-v_{2}\right|^{2 H^{\prime}-2}\left|v_{2}-v_{3}\right|^{2 H^{\prime}-2}\left|v_{3}-v_{4}+k\right|^{2 H^{\prime}-2} \\
& \times\left|v_{4}-v_{5}\right|^{2 H^{\prime}-2}\left|v_{5}-v_{6}\right|^{2 H^{\prime}-2}\left|v_{1}-v_{6}+k\right|^{2 H^{\prime}-2} d v_{1} \ldots d v_{6} .
\end{aligned}
$$

We put

$$
\begin{aligned}
\bar{A}_{3, N}^{(2)} & :=\frac{1}{N^{12 H^{\prime}-6}} \sum_{k=0}^{N-1}(N-k)\left|v_{3}-v_{4}+k\right|^{2 H^{\prime}-2}\left|v_{1}-v_{6}+k\right|^{2 H^{\prime}-2} \\
& =\frac{1}{N^{8 H^{\prime}-4}} \frac{1}{N} \sum_{k=0}^{N-1}\left(1-\frac{k}{N}\right)\left|\frac{v_{3}-v_{4}}{N}+\frac{k}{N}\right|^{2 H^{\prime}-2}\left|\frac{v_{1}-v_{6}}{N}+\frac{k}{N}\right|^{2 H^{\prime}-2}
\end{aligned}
$$

and we conclude easily by a Riemann sum convergence that

$$
N^{4 H} \bar{A}_{3, N}=N^{8 H^{\prime}-4} \bar{A}_{3, N} \underset{N \rightarrow \infty}{\longrightarrow} \int_{0}^{1}(1-x) x^{4 H^{\prime}-4} d x=\frac{1}{2 H-1}-\frac{1}{2 H}
$$


because the terms $\frac{v_{3}-v_{4}}{N}$ are negligible with respect to $\frac{k}{N}$ for large enough $k$. This implies that,

$$
N^{4 H} A_{3, N}^{(2)} \underset{N \rightarrow \infty}{\longrightarrow} \frac{d(H)^{6} a(H)^{6}}{H(2 H-1)}\left(C^{\prime}(H)\right)^{2}=\frac{H^{2}(2 H-1)^{2}}{8}\left(C^{\prime}(H)\right)^{2}
$$

where

$$
C^{\prime}(H)=\int_{[0,1]^{3}}\left|v_{1}-v_{2}\right|^{2 H^{\prime}-2}\left|v_{2}-v_{3}\right|^{2 H^{\prime}-2} d v_{1} d v_{2} d v_{3} .
$$

Now, we estimate the term $A_{2, N}^{(2)}$.

$$
\begin{aligned}
& \left\langle\left(f_{i, N},\left(f_{j, N} \otimes_{1} f_{j, N}\right) \otimes_{1} f_{j, N}\right\rangle_{L^{2}\left([0,1]^{2}\right)}\right. \\
= & d(H)^{4} a(H)^{4} \int_{I_{i}} \int_{I_{j}^{3}}\left|u_{1}-u_{2}\right|^{2 H^{\prime}-2}\left|u_{2}-u_{3}\right|^{2 H^{\prime}-2}\left|u_{3}-u_{4}\right|^{2 H^{\prime}-2}\left|u_{4}-u_{1}\right|^{2 H^{\prime}-2} d u_{1} \ldots d u_{4} \\
= & \frac{d(H)^{4} a(H)^{4}}{N^{8} H^{\prime}-4} \int_{[0,1]^{4}}\left|v_{1}-v_{2}+i-j\right|^{2 H^{\prime}-2}\left|v_{2}-v_{3}\right|^{2 H^{\prime}-2}\left|v_{3}-v_{4}\right|^{2 H^{\prime}-2}\left|v_{4}-v_{1}+j-i\right|^{2 H^{\prime}-2} d v_{1} \ldots d v_{4}
\end{aligned}
$$

Then

$$
\begin{aligned}
A_{2, N}^{(2)}= & \left\|f_{i, N}\right\|_{L^{2}\left([0,1]^{2}\right)}^{2} \frac{d(H)^{4} a(H)^{4}}{N^{8} H^{\prime}-4} \int_{[0,1]^{4}}\left|v_{2}-v_{3}\right|^{2 H^{\prime}-2}\left|v_{3}-v_{4}\right|^{2 H^{\prime}-2} \\
& \quad \times\left(\sum_{i, j=1}^{N}\left|v_{1}-v_{2}+i-j\right|^{2 H^{\prime}-2}\left|v_{4}-v_{1}+j-i\right|^{2 H^{\prime}-2}\right) d v_{1} \ldots d v_{4} \\
= & \frac{N^{-2 H}}{2} \frac{d(H)^{4} a(H)^{4}}{N^{8 H^{\prime}-4}} \int_{[0,1]^{4}}\left|v_{2}-v_{3}\right|^{2 H^{\prime}-2}\left|v_{3}-v_{4}\right|^{2 H^{\prime}-2} \\
& \times\left(2 \sum_{k=0}^{N-1}(N-k)\left|v_{1}-v_{2}+k\right|^{2 H^{\prime}-2}\left|v_{4}-v_{1}+k\right|^{2 H^{\prime}-2}\right) d v_{1} \ldots d v_{4} \\
= & N^{-2 H} \frac{d(H)^{4} a(H)^{4}}{N^{4 H^{\prime}-2}} \int_{[0,1]^{4}}\left|v_{2}-v_{3}\right|^{2 H^{\prime}-2}\left|v_{3}-v_{4}\right|^{2 H^{\prime}-2} \\
& \times\left(\frac{1}{N} \sum_{k=0}^{N-1}\left(1-\frac{k}{N}\right)\left|\frac{v_{1}-v_{2}}{N}+\frac{k}{N}\right|^{2 H^{\prime}-2}\left|\frac{v_{4}-v_{1}}{N}+\frac{k}{N}\right|^{2 H^{\prime}-2}\right) d v_{1} \ldots d v_{4} \\
= & \frac{d(H)^{4} a(H)^{4}}{N^{4 H}} \int_{[0,1]^{4}}\left|v_{2}-v_{3}\right|^{2 H^{\prime}-2}\left|v_{3}-v_{4}\right|^{2 H^{\prime}-2} \\
& \times\left(\frac{1}{N} \sum_{k=0}^{N-1}\left(1-\frac{k}{N}\right)\left|\frac{v_{1}-v_{2}}{N}+\frac{k}{N}\right|^{2 H^{\prime}-2}\left|\frac{v_{4}-v_{1}}{N}+\frac{k}{N}\right|^{2 H^{\prime}-2}\right) d v_{1} \ldots d v_{4} .
\end{aligned}
$$

We obtain that $N^{4 H} A_{2, N}^{(2)}$ converges as $N \rightarrow \infty$ to

$$
\begin{aligned}
& \left(\int_{0}^{1}(1-x) x^{4 H^{\prime}-4} d x\right)\left(d(H)^{4} a(H)^{4} \int_{[0,1]^{3}}\left|v_{2}-v_{3}\right|^{2 H^{\prime}-2}\left|v_{3}-v_{4}\right|^{2 H^{\prime}-2} d v_{2} \ldots d v_{4}\right) \\
= & \left(\frac{1}{2 H-1}-\frac{1}{2 H}\right)\left(d(H)^{4} a(H)^{4} \int_{[0,1]^{3}}\left|v_{2}-v_{3}\right|^{2 H^{\prime}-2}\left|v_{3}-v_{4}\right|^{2 H^{\prime}-2} d v_{2} \ldots d v_{4}\right)
\end{aligned}
$$


Thus

$$
N^{4 H} A_{2, N}^{(2)} \underset{N \rightarrow \infty}{\longrightarrow} C^{\prime}(H) \frac{H(2 H-1)}{8}
$$

From (18), (21) and (22), we obtain that

$$
N^{4 H} A_{N}^{(2)}=2\left(36 N^{4 H} A_{1, N}^{(2)}+144 N^{4 H} A_{2, N}^{(2)}+(24)^{2} N^{4 H} A_{3, N}^{(2)}\right)
$$

converges to $\left(9+36 C^{\prime}(H) H(2 H-1)+144\left[C^{\prime}(H) H(2 H-1)\right]^{2}\right):=C_{0}(H)$ as $N \longrightarrow \infty$.

Consequently

$$
\frac{N^{4 H} A_{N}^{(2)}}{C_{0}(H)} \underset{N \rightarrow \infty}{\longrightarrow} 1 .
$$

Estimation of the term $D_{N}^{(6)}$. Now, we study the convergence of $D_{N}^{(6)}$, using the symmetry property of every $f_{i, N}, i=0, \ldots, N-1$ on $[0,1]^{2}$, there exist positive combinatorial constants $c_{1}, c_{2}$ and $c_{3}$ such that

$$
\begin{aligned}
D_{N}^{(6)} & \left.\left.=\sum_{i, j=0}^{N-1} 6 !\left\langle\left(f_{i, N} \tilde{\otimes} f_{j, N}\right) \tilde{\otimes} f_{j, N}\right),\left(f_{j, N} \tilde{\otimes} f_{i, N}\right) \tilde{\otimes} f_{i, N}\right)\right\rangle_{L^{2}\left([0,1]^{6}\right)} \\
& =c_{1} \sum_{i, j=0}^{N-1}\left(\left\langle f_{i, N}, f_{j, N}\right\rangle_{L^{2}\left([0,1]^{2}\right)}\right)^{3} \\
& +c_{2} \sum_{i, j=0}^{N-1}\left\langle f_{i, N}, f_{j, N}\right\rangle_{L^{2}\left([0,1]^{2}\right)} \int_{[0,1]^{4}} f_{i, N}\left(x_{1}, x_{2}\right) f_{j, N}\left(x_{2}, x_{3}\right) f_{i, N}\left(x_{3}, x_{4}\right) f_{j, N}\left(x_{4}, x_{1}\right) d x_{1} \ldots d x_{4} \\
& +c_{3} \sum_{i, j=0}^{N-1} \int_{[0,1]^{6}} f_{i, N}\left(x_{1}, x_{2}\right) f_{j, N}\left(x_{2}, x_{3}\right) f_{i, N}\left(x_{3}, x_{4}\right) f_{j, N}\left(x_{4}, x_{5}\right) f_{i, N}\left(x_{5}, x_{6}\right) f_{j, N}\left(x_{6}, x_{1}\right) d x_{1} \ldots d x_{6} \\
& :=c_{1} D_{1, N}^{(6)}+c_{2} D_{2, N}^{(6)}+c_{3} D_{3, N}^{(6)} .
\end{aligned}
$$

By using the same argument as above, we have

$$
\begin{aligned}
D_{1, N}^{(6)} & =\sum_{i, j=0}^{N-1}\left(\left\langle f_{i, N}, f_{j, N}\right\rangle_{L^{2}\left([0,1]^{2}\right)}\right)^{3} \\
& =\frac{d(H)^{6} a(H)^{6}}{N^{6 H}} \sum_{i, j=0}^{N-1}\left(\int_{[0,1]^{2}}\left|x_{1}-x_{2}+i-j\right|^{2 H-2} d_{1} d x_{2}\right)^{3} \\
& =\frac{d(H)^{6} a(H)^{6}}{N^{6 H}} 2 \sum_{k=0}^{N-1}(N-k)\left(\int_{[0,1]^{2}}\left|x_{1}-x_{2}+k\right|^{2 H-2} d x_{1} d x_{2}\right)^{3} \\
& =\frac{2 d(H)^{6} a(H)^{6}}{N^{4}} \sum_{k=0}^{N-1} \frac{1}{N}\left(1-\frac{k}{N}\right)\left(\int_{[0,1]^{2}}\left|\frac{x_{1}-x_{2}}{N}+\frac{k}{N}\right|^{2 H-2}\right)^{3} .
\end{aligned}
$$


and clearly since $H<1$ we have

$$
\lim _{N \rightarrow \infty} N^{4 H} D_{1, N}^{(6)}=0 .
$$

By the same way, we obtain

$$
\begin{aligned}
D_{2, N}^{(6)}= & \sum_{i, j=0}^{N-1}\left\langle f_{i, N}, f_{j, N}\right\rangle_{L^{2}\left([0,1]^{2}\right)} \int_{[0,1]^{4}} f_{i, N}\left(x_{1}, x_{2}\right) f_{j, N}\left(x_{2}, x_{3}\right) f_{i, N}\left(x_{3}, x_{4}\right) f_{j, N}\left(x_{4}, x_{1}\right) d x_{1} \ldots d x_{4} \\
= & \sum_{i, j=0}^{N-1} N^{-4} d(H)^{4} a(H)^{4}\left(\left\langle f_{i, N}, f_{j, N}\right\rangle_{L^{2}\left([0,1]^{2}\right)}\right) \int_{[0,1]^{4}}\left|\frac{x_{1}-x_{2}}{N}+\frac{i-j}{N}\right|^{2 H^{\prime}-2} \\
& \quad \times\left|\frac{x_{3}-x_{2}}{N}+\frac{i-j}{N}\right|^{2 H^{\prime}-2}\left|\frac{x_{3}-x_{4}}{N}+\frac{i-j}{N}\right|^{2 H^{\prime}-2}\left|\frac{x_{1}-x_{4}}{N}+\frac{i-j}{N}\right|^{2 H^{\prime}-2} d x_{1} \ldots d x_{4} \\
= & \frac{2 d(H)^{6} a(H)^{6}}{N^{6}} \sum_{k=0}^{N-1}(N-k) \int_{[0,1]^{2}}\left|\frac{x_{5}-x_{6}}{N}+\frac{k}{N}\right|^{2 H-2} d x_{5} d x_{6} \int_{[0,1]^{4}}\left|\frac{x_{1}-x_{2}}{N}+\frac{k}{N}\right|^{2 H^{\prime}-2} \\
& \times\left|\frac{x_{3}-x_{2}}{N}+\frac{k}{N}\right|^{2 H^{\prime}-2}\left|\frac{x_{3}-x_{4}}{N}+\frac{k}{N}\right|^{2 H^{\prime}-2}\left|\frac{x_{1}-x_{4}}{N}+\frac{k}{N}\right|^{2 H^{\prime}-2} d x_{1} \ldots d x_{4} \\
= & \frac{2 d(H)^{6} a(H)^{6}}{N^{4}} \sum_{k=0}^{N-1} \frac{1}{N}\left(1-\frac{k}{N}\right) \int_{[0,1]^{2}}\left|\frac{x_{5}-x_{6}}{N}+\frac{k}{N}\right|^{2 H-2} d x_{5} d x_{6} \int_{[0,1]^{4}}\left|\frac{x_{1}-x_{2}}{N}+\frac{k}{N}\right|^{2 H^{\prime}-2} \\
& \times\left|\frac{x_{3}-x_{2}}{N}+\frac{k}{N}\right|^{2 H^{\prime}-2}\left|\frac{x_{3}-x_{4}}{N}+\frac{k}{N}\right|^{2 H^{\prime}-2}\left|\frac{x_{1}-x_{4}}{N}+\frac{k}{N}\right|^{2 H^{\prime}-2} d x_{1} \ldots d x_{4} .
\end{aligned}
$$

This implies that

$$
\lim _{N \rightarrow \infty} N^{4 H} D_{2, N}^{(6)}=0 .
$$

The same manner as in previous results, we have

$$
\begin{aligned}
D_{3, N}^{(6)}= & \sum_{i, j=0}^{N-1} \int_{[0,1]^{6}} f_{i, N}\left(x_{1}, x_{2}\right) f_{j, N}\left(x_{2}, x_{3}\right) f_{i, N}\left(x_{3}, x_{4}\right) f_{j, N}\left(x_{4}, x_{5}\right) f_{i, N}\left(x_{5}, x_{6}\right) f_{j, N}\left(x_{6}, x_{1}\right) d x_{1} \ldots d x_{6} \\
= & \frac{d(H)^{6} a(H)^{6}}{N^{6}} \sum_{i, j=0}^{N-1} \int_{[0,1]^{6}}\left|\frac{x_{1}-x_{2}}{N}+\frac{i-j}{N}\right|^{2 H^{\prime}-2}\left|\frac{x_{3}-x_{2}}{N}+\frac{i-j}{N}\right|^{2 H^{\prime}-2}\left|\frac{x_{3}-x_{4}}{N}+\frac{i-j}{N}\right|^{2 H^{\prime}-2} \\
& \times\left|\frac{x_{5}-x_{4}}{N}+\frac{i-j}{N}\right|^{2 H^{\prime}-2}\left|\frac{x_{5}-x_{6}}{N}+\frac{i-j}{N}\right|^{2 H^{\prime}-2}\left|\frac{x_{1}-x_{6}}{N}+\frac{i-j}{N}\right|^{2 H^{\prime}-2} d x_{1} \ldots d x_{6} \\
= & \frac{2 d(H)^{6} a(H)^{6}}{N^{4}} \sum_{k=0}^{N-1} \frac{1}{N}\left(1-\frac{k}{N}\right) \int_{[0,1]^{4}}\left|\frac{x_{1}-x_{2}}{N}+\frac{k}{N}\right|^{2 H^{\prime}-2}\left|\frac{x_{3}-x_{2}}{N}+\frac{k}{N}\right|^{2 H^{\prime}-2}\left|\frac{x_{3}-x_{4}}{N}+\frac{k}{N}\right|^{2 H^{\prime}-2} \\
& \times\left|\frac{x_{5}-x_{4}}{N}+\frac{k}{N}\right|^{2 H^{\prime}-2}\left|\frac{x_{5}-x_{6}}{N}+\frac{k}{N}\right|^{2 H^{\prime}-2}\left|\frac{x_{1}-x_{6}}{N}+\frac{k}{N}\right|^{2 H^{\prime}-2} d x_{1} \ldots d x_{6} .
\end{aligned}
$$

Hence

$$
\lim _{N \rightarrow \infty} N^{4 H} D_{3, N}^{(6)}=0 .
$$


Thus, from (25), (26) and (27), we obtain

$$
\lim _{N \rightarrow \infty} N^{4 H} D_{N}^{(6)}=0 .
$$

Estimation of the term $B_{N}^{(4)}$. Applying the same argument as in last part, there exist constants $c_{1}^{\prime}$ and $c_{2}^{\prime}$ such that

$$
\begin{aligned}
& \left\langle\widetilde{h_{i, N}}, \widetilde{h_{j, N}}\right\rangle_{L^{2}\left([0,1]^{4}\right)} \\
= & c_{1}^{\prime} \sum_{i, j=0}^{N-1}\left\langle f_{i, N}, f_{j, N}\right\rangle_{L^{2}\left([0,1]^{2}\right)} \int_{[0,1]^{4}} f_{i, N}\left(x_{1}, x_{2}\right) f_{j, N}\left(x_{2}, x_{3}\right) f_{i, N}\left(x_{3}, x_{4}\right) f_{j, N}\left(x_{4}, x_{1}\right) d x_{i} \\
+ & c_{2}^{\prime} \sum_{i, j=0}^{N-1} \int_{[0,1]^{6}} f_{i, N}\left(x_{1}, x_{2}\right) f_{j, N}\left(x_{2}, x_{3}\right) f_{i, N}\left(x_{3}, x_{4}\right) f_{j, N}\left(x_{4}, x_{5}\right) f_{i, N}\left(x_{5}, x_{6}\right) f_{j, N}\left(x_{6}, x_{1}\right) d x_{i} \\
= & c_{1}^{\prime} D_{2, N}^{(6)}+c_{2}^{\prime} D_{3, N}^{(6)} .
\end{aligned}
$$

The same terms as in the estimation of the sixth chaos kernel appear. Thus, from the convergences (26) and (27),

$$
\begin{aligned}
\lim _{N \rightarrow \infty} N^{4 H} B_{N}^{(4)} & =\lim _{N \rightarrow \infty} N^{4 H} \sum_{i, j=0}^{N-1}\left\langle\widetilde{h_{i, N}}, \widetilde{h_{j, N}}\right\rangle_{L^{2}\left([0,1]^{4}\right)} \\
& =\lim _{N \rightarrow \infty}\left(c_{1}^{\prime} N^{4 H} D_{2, N}^{(6)}+c_{2}^{\prime} N^{4 H} D_{3, N}^{(6)}\right)=0 .
\end{aligned}
$$

As a consequence of the convergences (24), (28) and (29), we have proved that for every $H>\frac{1}{2}$ and with the notation $\bar{C}(H)=\frac{C(H)^{2}}{C_{0}(H)}$,

$$
\frac{C(H)^{2}}{C_{0}(H)} N^{2-2 H} \mathbf{E}\left(V^{3, N}\right)^{2}=\mathbf{E}\left(\sqrt{\bar{C}(H)} N^{1-H} V^{3, N}\right)^{2} \underset{N \rightarrow \infty}{\longrightarrow} 1 .
$$

\subsection{Non-convergence to a Gaussian limit}

We prove that the normalized variation doesn't converge in distribution to the normal law. Of course this somehow superflu taking into account that in the next section we show a noncentral limit theorem for this statistics, but we found the calculations instructive to see why it does not converges to a Gaussian limit. Recall that by a result of [15] (Theorem 4 in this paper) a sequence $F_{N}=I_{q}\left(f_{N}\right)$ in the $q$ Wiener chaos with $\mathbb{E} F_{N}^{2} \rightarrow_{N} 1$ converges to the normal law $N(0,1)$ if and only if $\left\|D F_{N}\right\|_{L^{2}[0,1]}^{2}$ converges to $q$ in $L^{2}(\Omega)$ when $N \rightarrow \infty$. Here $D$ denotes the Malliavin derivative and if $f \in L^{2}\left([0, T]^{n}\right)$ is a symmetric function, we will use the following rule to differentiate in the Malliavin sense

$$
D_{t} I_{n}(f)=n I_{n-1}(f(\cdot, t)), \quad t \in[0,1] .
$$


We put

$$
T_{N}=\frac{\sqrt{\bar{C}(H)} N^{1-H}}{C(H) N^{1-3 H}} \sum_{i=0}^{N} I_{2}\left(g_{i, N}\right)=\frac{N^{2 H}}{\sqrt{C_{0}(H)}} \sum_{i=0}^{N} I_{2}\left(g_{i, N}\right)
$$

We derive $T_{N}$ in the Malliavin sense and we obtain $D_{t} T_{N}=\frac{2 N^{2 H}}{\sqrt{C_{0}(H)}} \sum_{i=0}^{N} I_{1}\left(g_{i, N}(., t)\right)$ and thus

$$
\begin{aligned}
\left\|D T_{N}\right\|_{L^{2}\left([0,1]^{2}\right)}^{2} & =\frac{4 N^{4 H}}{C_{0}(H)} \int_{0}^{1}\left(\sum_{i=0}^{N} I_{1}\left(g_{i, N}(., t)\right)\right)^{2} d t \\
& =\frac{4 N^{4 H}}{C_{0}(H)} \int_{0}^{1}\left(\sum_{i, j=0}^{N} I_{1}\left(g_{i, N}(., t)\right) I_{1}\left(g_{j, N}(., t)\right)\right) d t \\
& =\frac{4 N^{4 H}}{C_{0}(H)}\left(\sum_{i, j=0}^{N} \int_{0}^{1} I_{0}\left(g_{i, N} \otimes_{1} g_{j, N}\right) d t+\sum_{i, j=0}^{N} \int_{0}^{1} I_{2}\left(g_{i, N} \otimes_{0} g_{j, N}\right) d t\right) \\
& :=\frac{4 N^{4 H}}{C_{0}(H)}\left(J_{1, N}+J_{2, N}\right)
\end{aligned}
$$

where we denoted

$$
J_{1, N}=\sum_{i, j=0}^{N} \int_{0}^{1} I_{0}\left(g_{i, N} \otimes_{1} g_{j, N}\right) d t=\sum_{i, j=0}^{N}<g_{i, N}, g_{j, N}>_{L^{2}\left([0,1]^{2}\right)}=\frac{1}{2} A_{N}^{(2)} .
$$

From (24) we obtain

$$
\frac{4 N^{4 H}}{C_{0}(H)} J_{1, N} \underset{N \rightarrow \infty}{\longrightarrow} 2
$$

in $L^{2}(\Omega)$ because the term $A_{N}^{(2)}$ is deterministic. To prove that $\left\|D T_{N}\right\|_{L^{2}\left([0,1]^{2}\right)}^{2}$ not converges in $L^{2}(\Omega)$ to 2 , it is sufficient to show that

$$
\lim _{N \rightarrow \infty} \mathbf{E}\left(\frac{4 N^{4 H}}{C_{0}(H)} J_{2, N}\right)^{2}>0 .
$$

where $J_{2, N}=\sum_{i, j=0}^{N} \int_{0}^{1} I_{2}\left(g_{i, N}(., t) g_{j, N}(., t)\right) d t$. 
We calculate the mean square of this term.

$$
\begin{aligned}
\mathbf{E}\left(J_{2, N}\right)^{2}= & 2 \int_{[0,1]^{2}}\left(\sum_{i, j=0}^{N} \int_{0}^{1} I_{2}\left(g_{i, N}(r, t) g_{j, N}(s, t)\right) d t\right)^{2} d r d s \\
= & 2 \sum_{i, j, k, l=0}^{N} \int_{[0,1]^{4}} g_{i, N}(r, t) g_{j, N}(s, t) g_{k, N}(r, u) g_{l, N}(s, u) d r d s d t d u \\
\geq & \sum_{i, j, k, l=0}^{N} \int_{[0,1]^{4}} f_{i, N}(r, t) f_{j, N}(s, t) f_{k, N}(r, u) f_{l, N}(s, u) d r d s d t d u \\
= & 2 d(H)^{4} a(H)^{4} N^{-8 H} \sum_{i, j, k, l=0}^{N} \int_{I_{i}} \int_{I_{j}} \int_{I_{k}} \int_{I_{l}}(|r-t\|s-t\| r-u \| s-u|)^{2 H^{\prime}-2} d r d s d t d u \\
= & 2 d(H)^{4} a(H)^{4} N^{-8 H} \sum_{i, j, k, l=0}^{N} \int_{[0,1]^{4}} d r d s d t d u \\
& \frac{1}{N^{4}}\left(\left|\frac{r-t+i-j}{N}\left\|\frac{t-s+j-k}{N}\right\| \frac{s-u+k-l}{N} \| \frac{u-r+l-i}{N}\right|\right)^{2 H^{\prime}-2} .
\end{aligned}
$$

By using Riemann sums approximations, we obtain

$$
\lim _{N \rightarrow \infty} \mathbf{E}\left(N^{4 H} J_{2, N}\right)^{2} \geq 2 d(H)^{4} a(H)^{4} \int_{[0,1]^{4}} d x_{1} d x_{2} d x_{3} d x_{4}\left(\left|x_{1}-x_{2}\right|\left|x_{2}-x_{3}\right|\left|x_{3}-x_{4} \| x_{4}-x_{1}\right|\right)^{2 H^{\prime}-2}>0 .
$$

\section{The non-central limit theorem for the cubic variation of the Rosenblatt process}

Denote by $L_{t}$ the kernel of the Rosenblatt process

$$
L_{t}(x, y)=d(H) 1_{[0,1]}{ }^{\otimes 2}(x, y) \int_{0}^{t} \int_{0}^{t}\left(\int_{x \vee y}^{t} \partial_{1} K^{H^{\prime}}(s, x) \partial_{1} K^{H^{\prime}}(s, y) d s\right)
$$

and recall the notation

$$
f_{i, N}(x, y)=L_{\frac{i+1}{N}}^{(H)}(x, y)-L_{\frac{i}{N}}^{(H)}(x, y) .
$$

We proved in the previous section that the dominant term of the statistics $V^{3, N}$ which gives its normalization is

$$
C(H)^{-1} N^{3 H-1} \sum_{i=0}^{N-1} I_{2}\left(g_{i, N}\right)
$$

where

$$
\begin{aligned}
g_{i, N} & =6\left\|f_{i, N}\right\|_{L^{2}\left([0,1]^{2}\right)}^{2} f_{i, N}+24\left(f_{i, N} \otimes_{1} f_{i, N}\right) \otimes_{1} f_{i, N} \\
& =3 N^{-2 H} f_{i, N}+24\left(f_{i, N} \otimes_{1} f_{i, N}\right) \otimes_{1} f_{i, N}:=3 g_{i, N}^{(1)}+24 g_{i, N}^{(2)} .
\end{aligned}
$$

More precisely, it follows from the proof of Proposition 2 that

$$
\mathbf{E}\left[N^{1-H}\left(N^{3 H-1} \sum_{i=0}^{N-1} I_{2}\left(g_{i, N}^{(1)}\right)\right)\right]^{2}=N^{4 H} A_{1, N}^{(2)} \rightarrow_{N \rightarrow \infty} 1 / 8
$$


and

$$
\mathbf{E}\left[N^{1-H}\left(N^{3 H-1} \sum_{i=0}^{N-1} I_{2}\left(g_{i, N}^{(2)}\right)\right)\right]^{2}=N^{4 H} A_{3, N}^{(2)} \rightarrow_{N \rightarrow \infty} \frac{H^{2}(2 H-1)^{2}}{8}\left(C^{\prime}(H)\right)^{2} .
$$

Consequently, the limit of the sequence $V_{3, N}$ is the same as the limit of the sequence

$$
C(H)^{-1} N^{1-H} N^{3 H-1}\left(3 \sum_{i=0}^{N-1} I_{2}\left(g_{i, N}^{(1)}\right)+24 \sum_{i=0}^{N-1} I_{2}\left(g_{i, N}^{(2)}\right)\right) .
$$

We prove here our main result.

Theorem 3 The renormalized cubic variation statistics based on the Rosenblatt process $N^{1-H} V^{3, N}$ with $V^{3, N}$ given by (10) converges in $L^{2}(\Omega)$ as $N \rightarrow \infty$ to the Rosenblatt random variable $D(H) Z_{1}^{(H)}$ where $D(H)=C(H)^{-1}\left(3+24 d(H)^{2} a(H)^{2} C^{\prime}(H)\right)$.

Proof. To see the limit of $N^{1-H} V^{3, N}$ we need therefore to study the convergence of $N^{1-H}\left(N^{3 H-1} \sum_{i=0}^{N-1} I_{2}\left(g_{i, N}^{(1)}\right)\right)$ and of $N^{1-H}\left(N^{3 H-1} \sum_{i=0}^{N-1} I_{2}\left(g_{i, N}^{(2)}\right)\right)$.

Is easy to treat the first part. In fact we have

$$
N^{1-H} N^{3 H-1} \sum_{i=0}^{N-1} I_{2}\left(g_{i, N}^{(1)}\right)=N^{2 H} \sum_{i=0}^{N-1} N^{-2 H} I_{2}\left(f_{i, N}\right)=\sum_{i=0}^{N-1} I_{2}\left(f_{i, N}\right)=Z_{1}^{H}
$$

where $Z_{1}^{H}$ is a Rosenblatt random variable with selfsimilarity order $H$.

We find then the limit of the second part of the dominant term. We have

$$
N^{1-H} N^{3 H-1} \sum_{i=0}^{N-1} I_{2}\left(g_{i, N}^{(2)}\right)=N^{2 H} \sum_{i=0}^{N-1} I_{2}\left(\left(f_{i, N} \otimes_{1} f_{i, N}\right) \otimes_{1} f_{i, N}\right) .
$$

Let us denote by

$$
l^{H^{\prime}}(x, y, z, t):=\partial_{1} K^{H^{\prime}}(x, y) \partial_{1} K^{H^{\prime}}(z, t)
$$

and by

$$
\begin{gathered}
l_{0}^{H^{\prime}}(x, y, z):=\partial_{1} K^{H^{\prime}}(x, y) \partial_{1} K^{H^{\prime}}(x, z)=l^{H^{\prime}}(x, y, x, t) \\
l_{1}^{H^{\prime}}(x, y, z):=\partial_{1} K^{H^{\prime}}(x, z) \partial_{1} K^{H^{\prime}}(y, z)=l^{H^{\prime}}(x, z, y, z) .
\end{gathered}
$$

Using the relations (19) and (20) we get

$$
\begin{aligned}
& \left(\left(f_{i, N} \otimes_{1} f_{i, N}\right) \otimes_{1} f_{i, N}\right)\left(y_{1}, y_{2}\right) \\
= & d(H)^{3} a(H)^{2} 1_{\left[0, \frac{i}{N}\right]}^{\otimes 2}\left(y_{1}, y_{2}\right) \int_{I_{i}^{3}} d u_{1} d u_{2} d u_{3} l^{H^{\prime}}\left(u_{1}, y_{1}, u_{3}, y_{2}\right)\left[\left|u_{1}-u_{2}\right|\left|u_{2}-u_{3}\right|\right]^{2 H^{\prime}-2} \\
:= & b_{i, N}^{(1)}\left(y_{1}, y_{2}\right)+b_{i, N}^{(2)}\left(y_{1}, y_{2}\right)
\end{aligned}
$$


with

$b_{i, N}^{(1)}\left(y_{1}, y_{2}\right)=d(H)^{3} a(H)^{2} 1_{\left[0, \frac{i}{N}\right]}^{\otimes 2}\left(y_{1}, y_{2}\right) \int_{I_{i}^{3}} d u_{1} d u_{2} d u_{3} l^{H^{\prime}}\left(u_{1}, y_{1}, u_{3}, y_{2}\right)\left[\left|u_{1}-u_{2}\right|\left|u_{2}-u_{3}\right|\right]^{2 H^{\prime}-2}$

and

$$
\begin{aligned}
b_{i, N}^{(2)}\left(y_{1}, y_{2}\right) & =d(H)^{3} a(H)^{2} \\
& \times\left[1_{I_{i}}\left(y_{1}\right) 1_{\left[0, \frac{i}{N}\right]}\left(y_{2}\right) \int_{y_{1}}^{\frac{i+1}{N}} d u_{1} \int_{I_{i}^{2}} d u_{2} d u_{3} l^{H^{\prime}}\left(u_{1}, y_{1}, u_{3}, y_{2}\right)\left[\left|u_{1}-u_{2}\right|\left|u_{2}-u_{3}\right|\right]^{2 H^{\prime}-2}\right. \\
& +1_{\left[0, \frac{i}{N}\right]}\left(y_{1}\right) 1_{I_{i}}\left(y_{2}\right) \int_{I_{i}^{2}} d u_{1} d u_{2} \int_{y_{2}}^{\frac{i+1}{N}} d u_{3} l^{H^{\prime}}\left(u_{1}, y_{1}, u_{3}, y_{2}\right)\left[\left|u_{1}-u_{2}\right|\left|u_{2}-u_{3}\right|\right]^{2 H^{\prime}-2} \\
& \left.+1_{I_{i}^{2}}\left(y_{1}, y_{2}\right) \int_{y_{1}}^{\frac{i+1}{N}} d u_{1} \int_{I_{i}} d u_{2} \int_{y_{2}}^{\frac{i+1}{N}} d u_{3} l^{H^{\prime}}\left(u_{1}, y_{1}, u_{3}, y_{2}\right)\left[\left|u_{1}-u_{2}\right|\left|u_{2}-u_{3}\right|\right]^{2 H^{\prime}-2}\right] .
\end{aligned}
$$

We show that the $I_{2}\left(N^{2 H} \sum_{i=0}^{N-1} b_{i, N}^{(2)}\right)$ converges to zero in $L^{2}(\Omega)$ and it has no contribution to the limit. Indeed,

$$
\begin{aligned}
& \left.\mathbf{E}\left(I_{2}\left(N^{2 H} \sum_{i=0}^{N-1} b_{i, N}^{(2)}\right)\right)^{2}=2 N^{4 H} \int_{0}^{1} \int_{0}^{1} d y_{1} d y_{2}\left(\sum_{i=0}^{N-1} b_{i, N}^{2}\left(y_{1}, y_{2}\right)\right)\right)^{2} \\
& \leq 2 d(H)^{6} a(H)^{4} N^{4 H}\left(\sum_{i=0}^{N-1} \int_{0}^{1} \int_{0}^{1} d y_{1} d y_{2} \int_{I_{i}^{6}} d u_{1} d u_{1}^{\prime} d u_{2} d u_{2}^{\prime} d u_{3} d u_{3}^{\prime} 1_{\left[y_{1}, \frac{i+1}{N}\right]^{2}}\left(u_{1}, u_{1}^{\prime}\right)\right. \\
& \left.\times \quad 1_{\left[y_{2}, \frac{i+1}{N}\right]^{2}}\left(u_{3}, u_{3}^{\prime}\right) l_{1}^{H^{\prime}}\left(u_{1}, u_{1}^{\prime}, y_{1}\right) l_{1}^{H^{\prime}}\left(u_{3}, u_{3}^{\prime}, y_{2}\right)\left[\left|u_{1}-u_{2}\right|\left|u_{2}-u_{3}\right|\left|u_{1}^{\prime}-u_{2}^{\prime}\right|\left|u_{2}^{\prime}-u_{3}^{\prime}\right|\right]^{2 H^{\prime}-2}\right) \\
& \leq 2 d(H)^{6} a(H)^{4} N^{4 H}\left(\sum_{i=0}^{N-1} \int_{I_{i}^{6}} d u_{1} d u_{1}^{\prime} d u_{2} d u_{2}^{\prime} d u_{3} d u_{3}^{\prime}\left[\left|u_{1}-u_{2}\right|\left|u_{2}-u_{3}\left\|u_{1}^{\prime}-u_{2}^{\prime}\right\| u_{2}^{\prime}-u_{3}^{\prime}\right|\right]^{2 H^{\prime}-2}\right. \\
& \left.\times \quad \int_{0}^{u_{1} \wedge u_{1}^{\prime}} d y_{1} l_{1}^{H^{\prime}}\left(u_{1}, u_{1}^{\prime}, y_{1}\right) \int_{0}^{u_{3} \wedge u_{3}^{\prime}} d y_{2} l_{1}^{H^{\prime}}\left(u_{3}, u_{3}^{\prime}, y_{2}\right)\right) \\
& \leq 2 d(H)^{6} a(H)^{4} N^{4 H}\left(\sum_{i=0}^{N-1} \int_{I_{i}^{6}} d u_{1} d u_{1}^{\prime} d u_{2} d u_{2}^{\prime} d u_{3} d u_{3}^{\prime}\right. \\
& \left.\times \quad\left[\left|u_{1}-u_{2}\right|\left|u_{2}-u_{3}\right|\left|u_{1}^{\prime}-u_{2}^{\prime}\right|\left|u_{2}^{\prime}-u_{3}^{\prime}\right|\left|u_{1}-u_{1}^{\prime} \| u_{1}-u_{3}^{\prime}\right|\right]^{2 H^{\prime}-2}\right) \\
& \leq 2 d(H)^{6} a(H)^{4} N^{4 H} N N^{-6} N^{12-12 H^{\prime}}\left(\int_{[0,1]^{6}} d v_{1} d v_{1}^{\prime} d v_{2} d v_{2}^{\prime} d v_{3} d v_{3}^{\prime}\right. \\
& \left.\times \quad\left[\left|v_{1}-v_{2}\left\|v_{2}-v_{3}\right\| v_{1}^{\prime}-v_{2}^{\prime}\left\|v_{2}^{\prime}-v_{3}^{\prime}\right\| v_{1}-v_{1}^{\prime} \| v_{1}-v_{3}^{\prime}\right|\right]^{2 H^{\prime}-2}\right) \\
& \leq 2 c d(H)^{6} a(H)^{4} N^{1-2 H} \text {. }
\end{aligned}
$$


Combining with the fact that $H>\frac{1}{2}$, we conclude that

$$
I_{2}\left(N^{2 H} \sum_{i=0}^{N-1} b_{i, N}^{(2)}\right) \underset{N \rightarrow \infty}{\longrightarrow} 0 \quad \text { in } L^{2}(\Omega),
$$

and then we need to find the limit of

$$
\begin{aligned}
N^{2 H} \sum_{i=0}^{N-1} b_{i, N}^{(1)}= & d(H)^{3} a(H)^{2} N^{2 H} \sum_{i=0}^{N-1} 1_{\left[0, \frac{i}{N}\right]^{2}}\left(y_{1}, y_{2}\right) \\
& \times \int_{I_{i}^{3}} d u_{1} d u_{2} d u_{3} l^{H^{\prime}}\left(u_{1}, y_{1}, u_{3}, y_{2}\right)\left[\left|u_{1}-u_{2}\right|\left|u_{2}-u_{3}\right|\right]^{2 H^{\prime}-2} \\
= & d(H)^{3}(a(H))^{2} \sum_{i=0}^{N-1} 1_{\left[0, \frac{i}{N}\right]^{2}}\left(y_{1}, y_{2}\right) N^{-1} \\
& \times \int_{[0,1]^{3}} d v_{1} d v_{2} d v_{3} l^{H^{\prime}}\left(\frac{v_{1}+i}{N}, y_{1}, \frac{v_{3}+i}{N}, y_{2}\right)\left[\left|v_{1}-v_{2} \| v_{2}-v_{3}\right|\right]^{2 H^{\prime}-2}
\end{aligned}
$$

The last sequence has the same limit pointwise (for every $y_{1}, y_{2}$ ) as

$$
\begin{aligned}
d(H)^{3} a(H)^{2} & \int_{[0,1]^{3}} d v_{1} d v_{2} d v_{3}\left[\left|v_{1}-v_{2}\right|\left|v_{2}-v_{3}\right|\right]^{2 H^{\prime}-2} \\
& \times \sum_{i=0}^{N-1} 1_{\left[0, \frac{i}{N}\right]^{2}}\left(y_{1}, y_{2}\right) N^{-1} l^{H^{\prime}}\left(\frac{i}{N}, y_{1}, \frac{i}{N}, y_{2}\right) .
\end{aligned}
$$

This last term is a Riemann sum that converges to

$$
\begin{aligned}
& d(H)^{3} a(H)^{2} \int_{[0,1]^{3}} d v_{1} d v_{2} d v_{3}\left[\left|v_{1}-v_{2}\right|\left|v_{2}-v_{3}\right|\right]^{2 H^{\prime}-2} \int_{y_{1} \vee y_{2}}^{1} d x l^{H^{\prime}}\left(x, y_{1}, x, y_{2}\right) \\
= & d(H)^{3} a(H)^{2} C^{\prime}(H) \int_{y_{1} \vee y_{2}}^{1} d x \partial_{1} K^{H^{\prime}}\left(x, y_{1}\right) \partial_{1} K^{H^{\prime}}\left(x, y_{2}\right)=d(H)^{2} a(H)^{2} C^{\prime}(H) L_{1}^{(H)}(x, y)
\end{aligned}
$$

where $L_{1}^{(H)}$ is the standard kernel of the Rosenblatt process (6).

We need a Cauchy sequence argument as in [20] to conclude the proof. That is, we will show that the sequence $N^{2 H} \sum_{i=0}^{N-1} b_{i, N}^{(1)}$ (or equivalently $\left.N^{2 H} \sum_{i=0}^{N-1} g_{i, N}^{(2)}\right)$ is Cauchy in the Hilbert space $L^{2}\left([0,1]^{2}\right)$. This will imply that the sequence of random variable $I_{2}\left(N^{2 H} \sum_{i=0}^{N-1} g_{i, N}^{(2)}\right)$ is Cauchy, so convergent, in the space $L^{2}(\Omega)$ and it is easy to deduce that its limit coincides 
with the multiple integral of the pointwise limit of the kernel. We compute, for $M, N \geq 1$

$$
\begin{aligned}
& \left\|N^{2 H} \sum_{i=0}^{N-1} b_{i, N}^{(1)}-M^{2 H} \sum_{i=0}^{M-1} b_{i, M}^{(1)}\right\|_{L^{2}\left([0,1]^{2}\right)}^{2} \\
& =d(H)^{6} a(H)^{4}\left[N^{4 H} \sum_{i, j=0}^{N-1} \int_{I_{i}^{3}} \int_{I_{j}^{3}} d u_{1} d u_{2} d u_{3} d u_{1}^{\prime} d u_{2}^{\prime} d u_{3}^{\prime}\left[\left|u_{1}-u_{2}\right|\left|u_{2}-u_{3}\right|\left|u_{1}^{\prime}-u_{2}^{\prime}\right|\left|u_{2}^{\prime}-u_{3}^{\prime}\right|\right]^{2 H^{\prime}-2}\right. \\
& \times \int_{0}^{u_{1} \wedge u_{1}^{\prime}} d y_{1} \int_{0}^{u_{3} \wedge u_{3}^{\prime}} d y_{2} l^{H^{\prime}}\left(u_{1}, y_{1}, u_{3}, y_{2}\right) l^{H^{\prime}}\left(u_{1}^{\prime}, y_{1}, u_{3}^{\prime}, y_{2}\right) \\
& +M^{4 H} \sum_{i, j=0}^{M-1} \int_{I_{i}^{3}} \int_{I_{j}^{3}} d u_{1} d u_{2} d u_{3} d u_{1}^{\prime} d u_{2}^{\prime} d u_{3}^{\prime}\left[\left|u_{1}-u_{2}\right|\left|u_{2}-u_{3} \| u_{1}^{\prime}-u_{2}^{\prime}\right|\left|u_{2}^{\prime}-u_{3}^{\prime}\right|\right]^{2 H^{\prime}-2} \\
& \times \int_{0}^{u_{1} \wedge u_{1}^{\prime}} d y_{1} \int_{0}^{u_{3} \wedge u_{3}^{\prime}} d y_{2} l^{H^{\prime}}\left(u_{1}, y_{1}, u_{3}, y_{2}\right) l^{H^{\prime}}\left(u_{1}^{\prime}, y_{1}, u_{3}^{\prime}, y_{2}\right) \\
& \text { - } 2 N^{2 H} M^{2 H} \sum_{i=0}^{N-1} \sum_{j=0}^{M-1} \int_{I_{i}^{3}} \int_{I_{j}^{3}} d u_{1} d u_{2} d u_{3} d u_{1}^{\prime} d u_{2}^{\prime} d u_{3}^{\prime}\left[\left|u_{1}-u_{2}\left\|u_{2}-u_{3}\right\| u_{1}^{\prime}-u_{2}^{\prime}\right|\left|u_{2}^{\prime}-u_{3}^{\prime}\right|\right]^{2 H^{\prime}-2} \\
& \left.\times \int_{0}^{u_{1} \wedge u_{1}^{\prime}} d y_{1} \int_{0}^{u_{3} \wedge u_{3}^{\prime}} d y_{2} l^{H^{\prime}}\left(u_{1}, y_{1}, u_{3}, y_{2}\right) l^{H^{\prime}}\left(u_{1}^{\prime}, y_{1}, u_{3}^{\prime}, y_{2}\right)\right] \\
& =d(H)^{6} a(H)^{4}\left[N^{4 H} \sum_{i, j=0}^{N-1} \int_{I_{i}^{3}} \int_{I_{j}^{3}} d u_{1} d u_{2} d u_{3} d u_{1}^{\prime} d u_{2}^{\prime} d u_{3}^{\prime}\right. \\
& \times\left[\left|u_{1}-u_{2}\right|\left|u_{2}-u_{3}\left\|u_{1}^{\prime}-u_{2}^{\prime}|| u_{2}^{\prime}-u_{3}^{\prime}\right\| u_{1}-u_{1}^{\prime} \| u_{3}-u_{3}^{\prime}\right|\right]^{2 H^{\prime}-2} \\
& +M^{4 H} \sum_{i, j=0}^{M-1} \int_{I_{i}^{3}} \int_{I_{j}^{3}} d u_{1} d u_{2} d u_{3} d u_{1}^{\prime} d u_{2}^{\prime} d u_{3}^{\prime} \\
& \times\left[\left|u_{1}-u_{2}\right|\left|u_{2}-u_{3}\left\|u_{1}^{\prime}-u_{2}^{\prime}|| u_{2}^{\prime}-u_{3}^{\prime}\right\| u_{1}-u_{1}^{\prime} \| u_{3}-u_{3}^{\prime}\right|\right]^{2 H^{\prime}-2} \\
& -2 N^{2 H} M^{2 H} \sum_{i=0}^{N-1} \sum_{j=0}^{M-1} \int_{I_{i}^{3}} \int_{I_{j}^{3}} d u_{1} d u_{2} d u_{3} d u_{1}^{\prime} d u_{2}^{\prime} d u_{3}^{\prime} \\
& \left.\times\left[\left|u_{1}-u_{2}\right|\left|u_{2}-u_{3}\left\|u_{1}^{\prime}-u_{2}^{\prime}\right\| u_{2}^{\prime}-u_{3}^{\prime}\left\|u_{1}-u_{1}^{\prime}\right\| u_{3}-u_{3}^{\prime}\right|\right]^{2 H^{\prime}-2}\right]
\end{aligned}
$$


and this equal to

$$
\begin{aligned}
& d(H)^{6} a(H)^{4}\left[N^{-2 H} \sum_{i, j=0}^{N-1} \int_{[0,1]^{6}} d u_{1} d u_{2} d u_{3} d u_{1}^{\prime} d u_{2}^{\prime} d u_{3}^{\prime}\right. \\
& \times\left[\left|v_{1}-v_{2}\left\|v_{2}-v_{3}\right\| v_{1}^{\prime}-v_{2}^{\prime}\left\|v_{2}^{\prime}-v_{3}^{\prime}\right\| v_{1}-v_{1}^{\prime}+i-j \| v_{3}-v_{3}^{\prime}+i-j\right|\right]^{2 H^{\prime}-2} \\
+ & M^{-2 H} \sum_{i, j=0}^{M-1} \int_{[0,1]^{6}} d u_{1} d u_{2} d u_{3} d u_{1}^{\prime} d u_{2}^{\prime} d u_{3}^{\prime} \\
& \times\left[\left|v_{1}-v_{2}\left\|v_{2}-v_{3}\right\| v_{1}^{\prime}-v_{2}^{\prime}\left\|v_{2}^{\prime}-v_{3}^{\prime}\right\| v_{1}-v_{1}^{\prime}+i-j \| v_{3}-v_{3}^{\prime}+i-j\right|\right]^{2 H^{\prime}-2} \\
- & 2 N^{-1} M^{-1} \sum_{i=0}^{N-1} \sum_{j=0}^{M-1} \int_{[0,1]^{6}} d u_{1} d u_{2} d u_{3} d u_{1}^{\prime} d u_{2}^{\prime} d u_{3}^{\prime} \\
& \left.\times\left[\left|v_{1}-v_{2}\left\|v_{2}-v_{3}\right\| v_{1}^{\prime}-v_{2}^{\prime}\left\|v_{2}^{\prime}-v_{3}^{\prime}\right\| \frac{v_{1}}{N}-\frac{v_{1}^{\prime}}{M}+\frac{i}{N}-\frac{j}{M} \| \frac{v_{3}}{N}-\frac{v_{3}^{\prime}}{M}+\frac{i}{N}-\frac{j}{M}\right|\right]^{2 H^{\prime}-2}\right]
\end{aligned}
$$

The same way as in above this las term when $N \longrightarrow \infty$ and $N \longrightarrow \infty$ converges to

$$
\begin{aligned}
& d(H)^{6} a(H)^{4}\left[2 \int_{0}^{1}(1-x) x^{2 H-2} d x+2 \int_{0}^{1}(1-x) x^{2 H-2} d y+2 \int_{0}^{1} \int_{0}^{1}|x-y|^{2 H-2} d x d y\right] \\
& =\frac{1}{H(2 H-1)}+\frac{1}{H(2 H-1)}-\frac{2}{H(2 H-1)}=0 .
\end{aligned}
$$

We obtained that $\left\{N^{2 H} \sum_{i=0}^{N-1} b_{i, N}^{(1)}, N \geq 0\right\}$ is a Cauchy sequence and this completes the proof.

\section{References}

[1] J. Beran (1994): Statistics for Long-Memory Processes. Chapman and Hall.

[2] J.-C. Breton and I. Nourdin (2008): Error bounds on the non-normal approximation of Hermite power variations of fractional Brownian motion. Electronic Communications in Probability. 13, 482-493.

[3] P. Breuer and P. Major (1983): Central limit theorems for nonlinear functionals of Gaussian fields. J. Multivariate Analysis, 13 (3), 425-441.

[4] A. Chronopoulou, C.A. Tudor and F. Viens (2008): Self-similarity parameter estimation and reproduction property for non-Gaussian Hermite processes. Preprint.

[5] A. Chronopoulou, C.A. Tudor and F. Viens (2009): Variations and Hurst index estimation for a Rosenblatt process using longer filters. To appear in Electronic Journal of Statistics.

[6] J.F. Coeurjolly (2001): Estimating the parameters of a fractional Brownian motion by discrte variations of its sample paths. Statistical Inference for Stochastic Processes, 4, 199-227.

[7] R.L. Dobrushin and P. Major (1979): Non-central limit theorems for non-linear functionals of Gaussian fields. Z. Wahrscheinlichkeitstheorie verw. Gebiete, 50, 27-52. 
[8] P. Embrechts and M. Maejima (2002): Selfsimilar processes. Princeton University Press, Princeton, New York.

[9] J. Jacod and A.N. Shiryaev (2003): Limit theorems for stochastic processes. Second edition. Grundlehren der Mathematischen Wissenschaften [Fundamental Principles of Mathematical Sciences], 288, Springer.

[10] L. Giraitis and D. Surgailis (1985): CLT and other limit theorems for non-linear functionals of Gaussian processes. Z. Wahrsch. verw. Gebiete 70, 191-212.

[11] M. Maejima and C.A. Tudor (2007): Wiener integrals and a Non-Central Limit Theorem for Hermite processes, Stochastic Analysis and Applications, 25 (5), 1043-1056.

[12] I. Nourdin, D. Nualart and C.A Tudor (2007): Central and Non-Central Limit Theorems for weighted power variations of the fractional Brownian motion. Preprint.

[13] I. Nourdin, G. Peccati and A. Réveillac (2008): Multivariate normal approximation using Stein's method and Malliavin calculus. To appear in Annales I.H.P. .

[14] D. Nualart (2006): Malliavin Calculus and Related Topics. Second Edition. Springer.

[15] D. Nualart and S. Ortiz-Latorre (2008): Central limit theorems for multiple stochastic integrals and Malliavin calculus. Stochastic Processes and their Applications, 118, 614628.

[16] Yu. V. Prokhorov and V. Statulevicius, eds. (2006): Limit Theorems In Probability Theory. Springer.

[17] G. Samorodnitsky and M. Taqqu (1994): Stable Non-Gaussian random variables. Chapman and Hall, London.

[18] M. Taqqu (1975): Weak convergence to the fractional Brownian motion and to the Rosenblatt process. Z. Wahrscheinlichkeitstheorie verw. Gebiete, 31, 287-302.

[19] C.A. Tudor (2008): Analysis of the Rosenblatt process. ESAIM Probability and Statistics, 12, 230-257.

[20] C.A. Tudor and F. Viens (2009): Variations and estimators through Malliavin calculus. To appear in The Annals of Probability. 